\title{
Inc-SAMD14-4 can regulate expression of the COL1A1 and COL1A2 in human chondrocytes
}

\author{
Haibin Zhang ${ }^{1}$, Cheng Chen ${ }^{\text {Corresp., } 1}{ }^{1}$, Yinghong Cui ${ }^{2}$, Yuqing Li ${ }^{3}$, Zhaojun Wang ${ }^{4}$, Xinzhan Mao ${ }^{5}$, Pengcheng Dou ${ }^{5}$, \\ Yihan Li ${ }^{5}$, Chi Ma ${ }^{6}$ \\ 1 Department of Orthopedics, The N0.921 Hospital of the People's Liberation Army Joint Support Force, The Second Affiliated Hospital of Hunan Normal \\ University, Changsha, Hunan, China \\ 2 Department of Pharmaceutical Sciences, Hunan Normal University, changsha, Hunan, China \\ 3 Department of Orthopedics, Changsha central hospital, Changsha, Hunan, China \\ 4 Department of traumatology, Shanxi Fenyang Hospital, The Fenyang hospital of Shanxi Medical University, Fenyang, Shanxi, China \\ 5 Department of Orthopedics, The Second Xiangya Hospital of Central South University, Changsha, Hunan, China \\ 6 Department of Orthopedics, People's Hospital of Xiangxi Autonomous Prefecture, Jishou, Hunan, China \\ Corresponding Author: Cheng Chen \\ Email address: jc163yy@163.com
}

Osteoarthritis (OA) is the most common motor system disease in aging people, characterized by matrix degradation, chondrocyte death, and osteophyte formation. OA etiology is unclear, but long noncoding RNAs (IncRNAs) that participate in numerous pathological and physiological processes may be key regulators in the onset and development of OA. Because profiling of IncRNAs and their biological function in OA is not understood, we measured IncRNA and mRNA expression profiles using high-throughput microarray to study human knee OA. We identified 2,042 IncRNAs and 2,011 mRNAs that were significantly differentially expressed in OA compared to non-OA tissue ( $>2.0$ - or $<-2.0$-fold change; $p<0.5$ ), including 1,137 IncRNAs that were upregulated and 905 IncRNAs that were downregulated. Also, 1,386 mRNA were upregulated and 625 mRNAs were downregulated. QPCR was used to validate chip results. Gene Ontology analysis and the Kyoto Encyclopedia of Genes and Genomes was used to study the biological function enrichment of differentially expressed mRNA. Additionally, coding-non-coding gene coexpression (CNC) network construction was performed to explore the relevance of dysregulated IncRNAs and mRNAs. Finally, the gain/loss of function experiments of IncSAMD14-4 was implemented in IL-1ß-treated human chondrocytes. In genel, this study provides a preliminary database for further exploring IncRNA-related mechnisms in OA. 
2 Haibin Zhang ${ }^{1}$, Cheng Chen ${ }^{\#, 1}$, Yinghong Cui ${ }^{2}$, Yuqing $\mathrm{Li}^{3}$, Zhaojun Wang ${ }^{4}$, Xinzhan $\mathrm{Mao}^{5}$, Pengcheng Dou , 3 Yihan $\mathrm{Li}^{5}$, Chi $\mathrm{Ma}^{6}$,

4 1: Department of Orthopedics, The NO.921 Hospital of the People's Liberation Army Joint Support Force, The

5 Second Affiliated Hospital of Hunan Normal University, Changsha, Hunan 410011, PR China.

6 2: Department of Pharmaceutical Sciences, Medical College, Hunan Normal University, Changsha, Hunan 410013, 7 P.R. China.

8 3: Department of Orthopedics, Changsha central hospital, Changsha, Hunan 410003, PR China.

9 4: Department of traumatology, Shanxi Fenyang Hospital, The Fenyang hospital of Shanxi Medical University, 10 032200, PR China.

115 :Department of Orthopedics, The Second Xiangya Hospital of Central South University, Changsha, Hunan 410011, 12 PR China.

13 6:People's Hospital of Xiangxi Autonomous Prefecture, Department of Orthopedics, Jishou, Hunan, China

\section{4 \# Corresponding author}

15 Cheng Chen

16 Department of Orthopedics, The NO.921 Hospital of the People's Liberation Army Joint Support Force, The Second

17 Affiliated Hospital of Hunan Normal University, Changsha, Hunan 410003, PR China.

18 Tel: 13755132481

19 Email: jc163yy@163.com 


\section{ABSTRACT}

\section{Background}

31 Osteoarthritis (OA) is the most common motor system disease of aging people. It is characterized by matrix

32 degradation, chondrocyte death, and osteophyte formation. The etiology of OA is unclear, but long noncoding RNAs 33 (lncRNAs) that participate in numerous pathological and physiological processes may be key regulators of the onset

34 and development of OA. Here we report a new lncRNA (lnc-SAMD14-4) and explore its role and potential 35 involvement in osteoarthritis development.

\section{Methods}

37 Human knee cartilage tissue samples were obtained from patients. Then lncRNA and mRNA expression profiles were analyzed using Affymetrix human lncRNA chips. Gene ontology (GO) enrichment and KEGG pathway analysis of mRNAs were conducted to identify related biological function and pathologic pathways. Co-expression Additionally, real-time PCR was conducted to validate the expression pattern of some modulated lncRNA and

42 mRNA. To explore the role of IL-1 $\beta$ in osteoarthritis, human chondrocytes were treated with the factor and the modulation of lnc-SAMD14-4 was monitored through experimental gain or loss of function.

\section{Results}

45 A total of 2,042 lncRNAs and 2,011 mRNAs significantly differed in their expression in OA compared to non-OA 46 tissue. GO analysis was used to investigate the effect of all differentially expressed genes in the pathogenesis of OA.

47 In total, 27 pathways corresponded to up-regulated mRNAs and 94 pathways corresponded to down-regulated 48 mRNAs, as determined by KEGG pathway analysis. The results of qRT-PCR were consistent with microarray 49 profiling analysis. Remarkably, lnc-SAMD14-4 mediated upregulation of COL1A1 and COL1A2 in IL-1 $\beta$-treated 50 human chondrocytes.

\section{Conclusion}

52 This study provides a preliminary database for further exploration of lncRNA-related mechanisms relevant to OA development. It further revealed that lnc-SAMD14-4 may play a significant role in the development of OA.

\section{4}

55 


\section{Introduction}

58 Osteoarthritis $(\mathrm{OA})$ is an emerging global health burden prevalent among aging populations $[1,2]$. OA is caused by

59 cartilage matrix degradation, chondrocyte death, and osteophyte formation $[3,4]$. The pathology of OA is fairly well

60 understood and multiple therapies exist to treat patient symptoms. These include corticosteroids and nonsteroidal

61 anti-inflammatory drugs [5, 6], experimental stem cell therapies to treat specific forms of OA, and biologics to

62 address specific inflammatory mediators, such as cytokines. OA is also commonly treated with joint replacement

63 surgery. However, there is continued desire to find new therapeutic targets to improve osteoarthritis treatment.

64 Recent studies have evaluated the gene regulation capabilities of long noncoding ribonucleic acids (lncRNAs) in OA

65 development. LncRNAs are non-protein-coding transcripts (>200 nucleotides) that participate in numerous

66 pathological and physiological processes [7-9]. Some lncRNAs have been defined as key regulators in the onset and

67 development of OA [10-15]. For instance, overexpression of HOTAIR may contribute to OA development by

68 inducing chondrocyte apoptosis $[10,16]$. Fu et al. used microarray and bioinformatics to identify $4,714 \operatorname{lncRNAs}$

69 differentially expressed in knee cartilage from OA and non-OA patients [17]. Additionally, Liu et al. used

70 microarrays to identify 153 lncRNAs differentially expressed in OA patients and designated a specific lncRNA CIR

71 as critical to degradation of the chondrocyte matrix [14]. Differences in lncRNA expression are often attributed to

72 physiological processes that arise in laboratories using high-throughput screening. To address the lack of

73 information about the role of lncRNAs in the pathogenesis of OA, we used high-throughput microarray to measure

74 differentially expressed lncRNAs and mRNAs from the human knee cartilage of OA patients and non-OA patients. 


\section{Materials and methods}

\section{Specimen collection}

77 A Kellgren-Lawrence (KL) system was used to measure OA severity in patients [18]. OA cartilage (KL grades 3 or

78 4) was collected from 34 patients undergoing total knee joint replacement due to severe OA. Normal cartilage (KL 79 grades 0 ) was collected from 19 patients without history of rheumatoid arthritis or OA (who were being treated for 80 trauma, thromboangiities obliterans, osteosarcoma, or limb amputation). Informed consent was signed by all 81 patients and additional informed consent was obtained for publication of subject age and sex. This study was 82 approved by the Ethics Commission of the $163^{\text {rd }}$ Central Hospital of the Chinese People's Liberation Army (IRB.

83 [2015] 001). Patient data appears in Supplementary Table 1. Clinical specimens were divided for qPCR and for 84 array analyses.

85

\section{LncRNA and mRNA microarray analysis}

Quality testing, sample labeling, and hybridization to human WT lncRNA microarray (Affymetrix) were conducted by Oebiotech Co (Shanghai, China) (Supplementary Figure 1); their detailed protocol can be found in previous publications $[19,20]$. All RNA integrity number (RIN) values were $>7$, and 260:280 ratios were $>1.7$ (as measured by NanoDrop). The Genechip included 63,542 non-coding and 27,134 coding transcripts. Images were taken with an Affymetrix Scanner3000, and data normalization and probe filtering were conducted using an Affymetrix GeneChip Expression Console (version 4.0, Affymetrix). The lncRNA-mRNA expression between OA and non-OA groups was identified as $\mathrm{a} \geq 2.0$ fold-change (FC) for up-regulation or $\mathrm{a} \leq-2.0$ fold-change for down-regulation, with $\mathrm{p} \leq 0.05$. Afterwards, GO and KEGG analyses were used to identify roles of differentially expressed mRNAs with respect to GO terms or pathways. Their detailed definitions can be found in previous publications [21, 22]. A volcano plot and hierarchical clustering were created to show distinctions in gene expression patterns among samples. Finally, a coding-non-coding gene co-expression $(\mathrm{CNC})$ network was drawn using Cytoscape software.

The microarray data are available through the GEO database with the accession number GSE113825.

\section{RNA extraction and quantitative PCR}

A RNeasy Lipid Tissue Mini Kit (Qiagen, Germany) was used to extract total RNA from tissues according to the manufacturer's directions. A NanoDrop ND-1000 spectrophotometer was used to measure RNA quality and purity. Then SDS-PAGE was used to measure RNA integrity. Total RNA from samples was reverse transcribed into cDNA using an All-in-one First-Strand cDNA Synthesis Kit (GeneCopoeia Inc, Rockville, MD) as directed by the user 
103 manual. qPCR primers were designed and synthesized at the Shanghai Sangon Company (Supplementary Table 2);

104 qPCR was conducted using AceQ qPCR SYBR Green Master Mix (Vazyme Biotech Co, China) on a Bio-Rad CFX

105 ConnectTM Real-Time PCR detection system (Bio-Rad Germany) using $10 \mu \mathrm{l}$ SYBR Master Mix, $0.4 \mu \mathrm{l}$ forward

106 primer $(10 \mu \mathrm{M}), 0.4 \mu \mathrm{l}$ reverse primer $(10 \mu \mathrm{M}), 0.4 \mu \mathrm{l}$ ROX Reference Dye 1, $2 \mu \mathrm{l}$ cDNA, and $6.8 \mu \mathrm{l}$ MiliQ water.

107 The qPCR reaction conditions were as follows: pre-denaturation at $95^{\circ} \mathrm{C}$ for $5 \mathrm{~min}$, followed by $95{ }^{\circ} \mathrm{C}$ for $10 \mathrm{sec}$,

108 and $60{ }^{\circ} \mathrm{C}$ for $30 \mathrm{sec}$ for 40 cycles, and a final extension at $95^{\circ} \mathrm{C}$ for $15 \mathrm{sec}, 60^{\circ} \mathrm{C}$ for $60 \mathrm{sec}$, and $95^{\circ} \mathrm{C}$ for $15 \mathrm{sec}$.

109 The $2^{-\Delta \Delta C t}$ method was employed to assess gene expression, as described in previous studies [23]. Each experiment

110 was conducted in triplicate.

\section{Cell culture and treatment}

112 Human primary chondrocytes were procured from CHI Scientific, Inc (China). The cells were incubated in DMEM

113 (Gibco, USA) medium supplemented with 14\% FBS (Gibco, USA) in 6-well culture plates $\left(6 \times 10^{5} /\right.$ well). Cell

114 cultures were cultivated at $37^{\circ} \mathrm{C}$ in a $5 \% \mathrm{CO}_{2}$ incubator and the medium was changed every three days. All studies

115 were conducted using primary chondrocytes from passages $1-4$. Cells were treated either with or without $10 \mathrm{ng} / \mathrm{ml}$

116 human recombinant interleukin-1 beta (IL-1 $\beta$ ) (Sigma-Aldrich, St Louis, MO, Germany) for 12h, 24h and 48h.

\section{Cell transfection}

118 The overexpression vector, lnc-SAMD14-4(pcDNA3.1+- lnc-SAMD14-4), and blank vector,

119 pcDNA3.1(pcDNA3.1+- vector), were designed by and purchased from GeneChem Co., Ltd. (Shanghai, China). The

120 vectors were transfected into human primary chondrocytes using Lipofectamine 3000 (Invitrogen, USA). Three

121 targeting siRNA, including si-lnc-SAMD14-4 (1\#, 2\# and 3\#), and a control siRNA were designed and purchased

122 from Ribo Biotechnology Co. Ltd. (Guangdong, China). These siRNA (100nM/well) were transfected into human

123 primary chondrocytes to assess gain or loss of function of lnc-SAMD14-4.

\section{Statistical analysis}

125 Differences in expression of IncRNA-mRNA between OA and non-OA groups were assessed with a Student's $t$ test.

126 GO and KEGG pathways were assessed using Fisher's exact test. Other data were analyzed using one-way ANOVA

127 and multiple groups were compared using Bonferroni’s method. GraphPad Prism 6.0 (GraphPad Software San

128 Diego, CA) was applied for data analysis and drawing. Data are shown as means $\pm \mathrm{SD}$, and $p<0.05$ was considered

129 statistically significant.

130 Results

Peer) reviewing PDF | (2018:04:27804:5:1:NEW 11 Jul 2019) 


\section{Analysis of differential expression among IncRNAs-mRNAs in OA}

132 We found 2,042 lncRNAs differed significantly in expression levels between OA and non-OA tissue

133 (Supplementary Table 3). The most upregulated lncRNA was lnc-SAMD14-4 and the most downregulated lncRNA

134 was lnc-MSMP-2. The 15 most dysregulated (either down or up) lncRNAs are listed in Table 1.

135 mRNA expression data revealed dysregulation of 2,011 mRNAs in OA cartilage tissue, as compared to non-OA

136 cartilage tissue; it was found that 1,386 mRNA were upregulated and 625 mRNAs were downregulated

137 (Supplementary Table 4). Among these mRNAs, the most upregulated protein coding RNA was MMP-13, which is 138 responsible for regulating articular cartilage degradation [24]. MYOC was the most strongly down-regulated. The

139 top 15 upregulated and downregulated mRNAs appear in Table 2. Data on differentially expressed genes (including

140 lncRNA and mRNA) appear in Fig. 1A-C.

141 Gene Ontology (GO) analysis and Kyoto Encyclopedia of Genes and Genomes (KEGG) analysis of

\section{2 dysregulated mRNAs}

143 The most enriched GOs targeted by upregulated mRNAs were extracellular matrix organization (GO:0030198),

144 extracellular exosome (GO:0070062), and collagen binding (GO:0005518) in biological process, cellular process,

145 and molecular function (Fig. 2A-C, Supplementary Table 5-7). Protein stabilization (GO:0050821), cilium

146 (GO:0005929), and heparan sulfate proteoglycan binding (GO:0043395) were the most enriched GO pathways

147 associated with downregulated transcripts (Fig. 2D-F, Supplementary Table 8-10).

148 We identified 27 pathways with upregulated mRNAs and the most enriched pathway was cytochrome P450

149 (Supplementary Table 11). Additionally, 94 pathways corresponded to downregulated mRNAs, according to KEGG 150 pathway analysis, and the top enriched pathway was focal adhesion (Supplementary Table 12). The top 20 pathways 151 enriched by downregulated and upregulated transcripts appear in Fig 3A and B.

\section{LncRNA-mRNA co-expression network}

153 Co-expression networks are of biological interest because co-expressed genes are controlled by the same

154 transcriptional regulatory program, are functionally related, or are members of the same pathway or protein complex

$155[25,26]$. It has been confirmed that apoptosis is involved in OA pathogenesis [27-30], and that the PI3K-Akt

156 signaling pathway is important for apoptosis [31]. We analyzed the 74 dysregulated mRNAs found to be enriched in

157 the PI3K-Akt signaling pathway (Supplementary Table 13) and identified 1,974 correlations for lncRNA-mRNA

158 interaction pairs that satisfy $\mathrm{p}<0.01$ and Pearson correlation coefficients $>0.95$ (Supplementary Table 14). Then, the

Peer) reviewing PDF | (2018:04:27804:5:1:NEW 11 Jul 2019) 
159 top 500 of 1,974 lncRNA-mRNA pairs were used to draw the network (Supplementary Table 15). Of the top 500 160 lncRNA-mRNA pairs, which included 57 mRNAs and 363 lncRNAs, 362 pairs had a positive association; the rest

161 were negatively associated. It appears that a single mRNA may pair with multiple lncRNAs while most lncRNAs

162 may only pair with a single mRNA (Fig. 4). Some lncRNA-mRNA pairs included the most dysregulated mRNA and 163 lncRNA. For example, COL1A1 (mRNA, FC=175.43) and COL1A2 (mRNA, FC=85.32) were strongly associated

164 with lnc-SAMD14-4 (lncRNA, FC= 86.11). All lncRNA-mRNA co-expressed pairs had Pearson correlation 165 coefficients $>0.98$

\section{Verification of IncRNAs and mRNAs expression by qRT-PCR}

167 To confirm microarray profiling analysis, four lncRNAs (lnc-SAMD14-4, lnc-MARCKS-7, lnc-MSMP-2 and lnc-

168 NPVF-4) and four mRNAs (SERPINF1, COL1A1, MYOC and PAK3) were selected and measured using qRT-PCR 169 in 53 clinical specimens (34 OA and 19 non-OA specimens). The results from the qRT-PCR agreed with chip 170 profiling analysis (Fig. 5).

\section{Prediction of novel IncRNA targeting}

172 We studied the most dysregulated lncRNA, lnc-SAMD14-4, for its potential function in OA development. To 173 analyze the gene coding-potential of lnc-SAMD14-4, the open-reading frames (ORFs) and codon-substitution 174 frequency (CSF) were measured according to former research protocol [14, 32]. The ORF Finder (National Center 175 for Biotechnology Information) failed to predict ORFs more than 300nt. Furthermore, BLAST search of all the 176 positive-sense strand ORFs, which are marked by “+” in Supplementary Figure 2, yielded no highly homologous 177 protein among short peptides. Alternatively, PhyloCSF analysis of lnc-SAMD14-4 was negative (Supplementary

178 Figure 3). These results confirmed that lnc-SAMD14-4 does not encode any functional protein product.

COL1A1, COL1A2 and lnc-SAMD14-4 were highly expressed in the IL-1ß-treated human primary

181 According to our co-expression network analysis, COL1A1 and COL1A2 were strongly associated with lnc-

182 SAMD14-4. Thus, we speculated that lnc-SAMD14-4 may contribute to OA by interacting with COL1A1 and 183 COL1A2. Previous studies have used human primary chondrocytes treated with IL-1 $\beta$-treated to model the process 184 of human osteoarthritis $[17,33]$. We determined expression levels of COL1A1, COL1A2 and lnc-SAMD14-4 by qPCR. We found that $C O L 1 A 1$ and $C O L 1 A 2$ were upregulated after human chondrocytes were treated with IL-1 $\beta$ for $24 \mathrm{~h}$ and $48 \mathrm{~h}$. Furthermore, upregulation of lnc-SAMD14-4 with IL-1 $\beta$ treatment was time-dependent (Fig. 6). 
187 These results suggest a positive correlation between lnc-SAMD14-4 and COL1A1, COL1A2 expression induced by

188 IL-1 $\beta$ treatment. Hence, human primary chondrocytes were treated either with or without IL-1 $\beta$ for $48 \mathrm{~h}$ in the

189 follow-up experiments.

190 Inc-SAMD14-4 was involved in IL-1 $\beta$-induced COL1A1 and COL1A2 expression in human primary

191 chondrocytes

192 To further explore the function of lnc-SAMD14-4 in eliciting IL-1 $\beta$-induced COL1A1 and COL1A2 expression, we 193 performed gain/loss of function experiments. We confirmed that lnc-SAMD14-4 expression in human primary 194 chondrocytes increased after pcDNA3.1+- lnc-SAMD14-4 transfection (Supplementary Figure 4). Additionally, as

195 shown in Figure 7, lnc-SAMD14-4 overexpression promoted expression of COL1A1 and COL1A2 in the human 196 primary chondrocytes, regardless of treatment with IL-1 $\beta(p<0.05)$.

197 To explore the knockdown effects of lnc-SAMD14-4 on COL1A1 and COL1A2, we designed and transfected three

198 independent si-lnc-SAMD14-4 (1\#, 2\# and 3\#) in human primary chondrocytes. The strongest silencing effects were 199 observed in si-lnc-SAMD14-4 (2\#), which was used for silencing in subsequent experiments (Supplementary Figure

200 5). The knockdown of lnc-SAMD14-4 significantly decreased the expression of COL1A1 and COL1A2 in human

201 primary chondrocytes, regardless of treatment with IL-1 $\beta$-treatment (Figure 8).

\section{Discussion}

203 To our knowledge, there has been some study focusing on the lncRNA expression profiles in osteoarthritis (OA).

204 For instance, Fu et al. identified 4,714 lncRNAs and Liu et al. identified 152 lncRNAs that were differentially

205 expressed in OA cartilage as compared to non-OA cartilage Pearson's group treated primary chondrocytes isolated

206 from hip articular cartilage with IL-1 $\beta$ to stimulate OA. This led them to identify lncRNAs related to the

207 inflammatory response in human hip OA cartilage $[11,17]$. However, there was much left to learn regarding the

208 roles of lncRNAs in osteoarthritis.

209 In the present study, we analyzed human knee cartilage from OA and non-OA patients by measuring expression

210 profiles of lncRNAs and mRNAs using high-throughput microarray and bioinformatics. For this, we used a

211 standards filter of $\mathrm{FC} \geq 2.0, p<0.05$. An important point to note is that our work used Affymetrix and previous

212 studies used Arraystar, which is a different platform. This was problematic when comparing lncRNA data.

213 Using these chips, we identified 2,042 dysregulated lncRNAs and 2,011 differentially expressed mRNAs in OA

214 samples as compared to non-OA tissues. These included COL1A1, COL1A2, MMP-13, MMP-9, and TNFAIP6, 
215 which are associated with cartilage biology and OA [34-38]. Excluding MMP13 mRNA from the study (which has 216 already been linked to chondrocyte biological processes and osteoarthritis [24]), studies with the top 4 dysregulated

217 IncRNAs and mRNAs verified that qRT-PCR data for 53 specimens (34 OA and 19 normal specimens) were

218 consistent with chip analysis.

219 Next, GO analysis and pathway analysis analyzed the biological function enrichment of differentially expressed

220 mRNA. For instance, the most enriched GO terms targeted by up-regulated mRNAs were extracellular matrix

221 organization (GO:0030198), extracellular exosome (GO:0070062), and collagen binding (GO:0005518) in

222 biological processes, cellular processes, and molecular function. Functions of the extracellular matrix and collagen

223 binding have been accepted as important to the onset and progression of OA [39, 40].

224 A lncRNA-mRNA co-expression network was constructed to identify associations between lncRNAs and mRNAs.

225 The fundamental principle of co-expression network analysis is that opposite or identical expression pattern of

226 mRNAs and lncRNAs indicate some interaction between the lncRNA-mRNA. We noticed that most dysregulated

227 IncRNA or mRNA made lncRNA-mRNA pairs. Among these, lnc-SAMD14-4 (lncRNA, FC= 86.11) was strongly

228 associated with COL1A1 (mRNA, FC=175.43) and COL1A2 (mRNA, FC=85.32). Type I collagen is a triple helix

229 consisting of two pro-alpha1(I) chains encoded by two COL1A1 genes and one pro-alpha2(I) chain encoded by one

230 COL1A2 gene. Type I collagen is the most abundant collagen of the human body present in scar tissue and it is also

231 regarded as the end product when tissue heals [41]. It is reported that Type I collagen is rare in normal chondrocytes,

232 however, higher levels of Type I collagen was detected in osteoarthritis cartilage, particularly in late stages of

233 osteoarthritis [42].

234 It is reasonable to suggest that lnc-SAMD14-4 may promote expression of the COL1A1 gene and COL1A2 gene,

235 thereby contributing to the pathogenesis of OA. To verify this hypothesis, Il-1 $\beta$-treated human chondrocytes were

236 used to imitate the genetic change associated with osteoarthritis development, as uncovered in previous studies [17,

237 43]. Our study suggested a positive correlation between expression of lnc-SAMD14-4 and COL1A1 gene and

238 COL1A2 gene in Il-1 $\beta$-treated human chondrocytes. This is consistent with expression patterns found in cartilage

239 from osteoarthritis patients. Thus, lnc-SAMD14-4 may play a key role in the pathogenesis of osteoarthritis by

240 promoting the expression of the COL1A1 and COL1A2 genes.

241 Age is the highest risk factor for the pathogenesis of osteoarthritis. This link between aging and OA is complex, but

242 there are some common physical mechanisms in aging and OA. One limitation of this study is the lack of age-

Peer] reviewing PDF | (2018:04:27804:5:1:NEW 11 Jul 2019) 
243 matched patients. In clinical practice, the age of OA patients was usually older than the age of non-OA patients.

244 However, some of the most dysregulated mRNAs in our study, including MMP-13 and POSTN, were OA-related

245 [44-46], which suggests that the differences observed in our arrays were at least in part a consequence of OA.

246 Therefore, further research should be performed with age-matched patients or a more ideal model in the future.

\section{Conclusion}

248 Taken together, the Affymetrix chips and bioinformatics methods were combined to explore differential expression

249 of lncRNAs from OA articular cartilage and non-OA articular cartilage. This study revealed that lnc-SAMD14-4

250 may upregulate the COL1A1 and COL1A2 genes, contributing to the pathogenesis of OA. This provides a potential

251 therapeutic target for treatment.

252

\section{Reference}

[1] S.J. Nho, S.M. Kymes, J.J. Callaghan, D.T. Felson, The burden of hip osteoarthritis in the United States: epidemiologic and economic considerations, The Journal of the American Academy of Orthopaedic Surgeons, 21 Suppl 1 (2013) S1-6.

257 [2] R.F. Loeser, Aging processes and the development of osteoarthritis, Current opinion in rheumatology, 25 (2013)

258 108-113.

259 [3] T. Hayami, M. Pickarski, Y. Zhuo, G.A. Wesolowski, G.A. Rodan, L.T. Duong, Characterization of articular 260 cartilage and subchondral bone changes in the rat anterior cruciate ligament transection and meniscectomized models of osteoarthritis, Bone, 38 (2006) 234-243.

[4] B. Carames, N. Taniguchi, S. Otsuki, F.J. Blanco, M. Lotz, Autophagy is a protective mechanism in normal cartilage, and its aging-related loss is linked with cell death and osteoarthritis, Arthritis Rheum, 62 (2010) $791-801$.

[5] J. Bohensky, S.P. Terkhorn, T.A. Freeman, C.S. Adams, J.A. Garcia, I.M. Shapiro, V. Srinivas, Regulation of autophagy in human and murine cartilage: hypoxia-inducible factor 2 suppresses chondrocyte autophagy, Arthritis Rheum, 60 (2009) 1406-1415.

[6] N. Bellamy, J. Campbell, V. Robinson, T. Gee, R. Bourne, G. Wells, Intraarticular corticosteroid for treatment of osteoarthritis of the knee, The Cochrane database of systematic reviews, (2006) CD005328.

[7] M. Guttman, J.L. Rinn, Modular regulatory principles of large non-coding RNAs, Nature, 482 (2012) 339-346.

[8] K.C. Wang, H.Y. Chang, Molecular Mechanisms of Long Noncoding RNAs, Molecular cell, 43 (2011) 904-914.

[9] A.F. Palazzo, E.S. Lee, Non-coding RNA: what is functional and what is junk?, Frontiers in genetics, 6 (2015) 2.

[10] C. Zhang, P. Wang, P. Jiang, Y. Lv, C. Dong, X. Dai, L. Tan, Z. Wang, Upregulation of IncRNA HOTAIR contributes to IL-1beta-induced MMP overexpression and chondrocytes apoptosis in temporomandibular joint osteoarthritis, Gene, 586 (2016) 248-253.

[11] M.J. Pearson, A.M. Philp, J.A. Heward, B.T. Roux, D.A. Walsh, E.T. Davis, M.A. Lindsay, S.W. Jones, Long Intergenic Noncoding RNAs Mediate the Human Chondrocyte Inflammatory Response and Are Differentially Expressed in Osteoarthritis Cartilage, Arthritis Rheumatol, 68 (2016) 845-856.

[12] W. Su, W. Xie, Q. Shang, B. Su, The Long Noncoding RNA MEG3 Is Downregulated and Inversely Associated with VEGF Levels in Osteoarthritis, BioMed Research International, 2015 (2015) 1-5.

[13] J. Song, C. Ahn, C.H. Chun, E.J. Jin, A long non-coding RNA, GAS5, plays a critical role in the regulation of miR21 during osteoarthritis, Journal of orthopaedic research : official publication of the Orthopaedic Research Society, 32 (2014) 1628-1635.

[14] Q. Liu, X. Zhang, L. Dai, X. Hu, J. Zhu, L. Li, C. Zhou, Y. Ao, Long noncoding RNA related to cartilage injury promotes chondrocyte extracellular matrix degradation in osteoarthritis, Arthritis Rheumatol, 66 (2014) 969-978.

[15] E. Steck, S. Boeuf, J. Gabler, N. Werth, P. Schnatzer, S. Diederichs, W. Richter, Regulation of H19 and its

PeerJ reviewing PDF | (2018:04:27804:5:1:NEW 11 Jul 2019) 
286

287

288

289

290

291

292

293

294

295

296

297

298

299

300

301

302

303

304

305

306

307

308

309

310

311

312

313

314

315

316

317

318

319

320

321

322

323

324

325

326

327

328

329

330

331

332

333

334

335

336

337

338

encoded microRNA-675 in osteoarthritis and under anabolic and catabolic in vitro conditions, J Mol Med (Berl), 90 (2012) 1185-1195.

[16] D. Xing, J.Q. Liang, Y. Li, J. Lu, H.B. Jia, L.Y. Xu, X.L. Ma, Identification of Long Noncoding RNA Associated with Osteoarthritis in Humans, Orthopaedic Surgery, 6 (2014) 288-293.

[17] M. Fu, G. Huang, Z. Zhang, J. Liu, Z. Huang, B. Yu, F. Meng, Expression profile of long noncoding RNAs in cartilage from knee osteoarthritis patients, Osteoarthritis and cartilage / OARS, Osteoarthritis Research Society, 23 (2015) 423-432.

[18] L.J. Kellgren JH, Radiological Assessment of Osteo-Arthrosis, Annals of the Rheumatic Diseases, 16 (1957) 494502 .

[19] S. Lightfoot, Quantitation comparison of total RNA using the Agilent 2100 bioanalyzer, ribo- green analysis and UV spectrometry.

[20] O. Mueller, K. Hahnenberger, M. Dittmann, H. Yee, R. Dubrow, R. Nagle, D. Ilsley, A microfluidic system for high-speed reproducible DNA sizing and quantitation, Electrophoresis, 21 (2015) 128-134.

[21] T. Huang, L. Chen, Y.D. Cai, K.C. Chou, Classification and analysis of regulatory pathways using graph property, biochemical and physicochemical property, and functional property, PloS one, 6 (2011) e25297.

[22] H. Tao, Z. Jian, X. Zhong-Ping, H. Le-Le, C. Lei, S. Jian-Lin, Z. Lei, K. Xiang-Yin, C. Yu-Dong, C. Kuo-Chen, Deciphering the effects of gene deletion on yeast longevity using network and machine learning approaches, Biochimie, 94 (2012) 1017-1025.

[23] K.J. Livak, T.D. Schmittgen, Analysis of relative gene expression data using real-time quantitative PCR and the 2(-Delta Delta C(T)) Method, Methods, 25 (2001) 402-408.

[24] A.J. Fosang, K. Last, V. Knäuper, G. Murphy, P.J. Neame, Degradation of cartilage aggrecan by collagenase-3 (MMP-13), FEBS letters, 380 (1996) 17-20.

[25] M.T. Weirauch, Gene Coexpression Networks for the Analysis of DNA Microarray Data, 2011.

[26] J.M. Stuart, E. Segal, D. Koller, S.K. Kim, A Gene-Coexpression Network for Global Discovery of Conserved Genetic Modules, Science, 302 (2003) 249.

[27] H.B. Zhang, Y. Zhang, C. Chen, Y.Q. Li, C. Ma, Z.J. Wang, Pioglitazone inhibits advanced glycation end productinduced matrix metalloproteinases and apoptosis by suppressing the activation of MAPK and NF-KB, Apoptosis : an international journal on programmed cell death, 21 (2016) 1082-1093.

[28] H.S. Hwang, H.A. Kim, Chondrocyte Apoptosis in the Pathogenesis of Osteoarthritis, International journal of molecular sciences, 16 (2015) 26035-26054.

[29] C.M.R. Andrew T. Pennock, Bryan C. Emmerson,, a.D.A. Frederick L. Harwood, Role of Apoptotic and MatrixDegrading Genes in Articular Cartilage and Meniscus of Mature and Aged Rabbits During Development of Osteoarthritis, ARTHRITIS \& RHEUMATISM, 56 (2007).

[30] H.A. Kim, F.J. Blanco, Cell death and apoptosis in osteoarthritic cartilage, Current drug targets, 8 (2007) 333345.

[31] T.F. Franke, C.P. Hornik, L. Segev, G.A. Shostak, C. Sugimoto, PI3K/Akt and apoptosis: size matters, Oncogene, 22 (2003) 8983-8998.

[32] L. Qu, J. Ding, C. Chen, Z.J. Wu, B. Liu, Y. Gao, W. Chen, F. Liu, W. Sun, X.F. Li, X. Wang, Y. Wang, Z.Y. Xu, L. Gao, Q. Yang, B. Xu, Y.M. Li, Z.Y. Fang, Z.P. Xu, Y. Bao, D.S. Wu, X. Miao, H.Y. Sun, Y.H. Sun, H.Y. Wang, L.H. Wang, Exosome-Transmitted IncARSR Promotes Sunitinib Resistance in Renal Cancer by Acting as a Competing Endogenous RNA, Cancer cell, 29 (2016) 653-668.

[33] M.B. Goldring, J. Birkhead, L.J. Sandell, T. Kimura, S.M. Krane, Interleukin 1 suppresses expression of cartilagespecific types II and IX collagens and increases types I and III collagens in human chondrocytes, The Journal of clinical investigation, 82 (1988) 2026-2037.

[34] A.-M. Freyria, F. Mallein-Gerin, Chondrocytes or adult stem cells for cartilage repair: The indisputable role of growth factors, Injury, 43 (2012) 259-265.

[35] M. Attur, Q. Yang, K. Shimada, Y. Tachida, H. Nagase, P. Mignatti, L. Statman, G. Palmer, T. Kirsch, F. Beier, Elevated expression of periostin in human osteoarthritic cartilage and its potential role in matrix degradation via matrix metalloproteinase-13, Faseb Journal, 23 (2015) A135-A136.

[36] A. Al-Sabah, P. Stadnik, S.J. Gilbert, V.C. Duance, E.J. Blain, Importance of reference gene selection for articular cartilage mechanobiology studies, Osteoarthritis \& Cartilage, 24 (2016) 719-730.

[37] S. Roberts, J. Menage, L.J. Sandell, E.H. Evans, J.B. Richardson, Immunohistochemical study of collagen types I and II and procollagen IIA in human cartilage repair tissue following autologous chondrocyte implantation, The

Peer] reviewing PDF | (2018:04:27804:5:1:NEW 11 Jul 2019) 
339

340

341

342

343

344

345

346

347

348

349

350

351

352

353

354

355

356

357

358

359

360

361

362

363

364

365

366

367

368

369

370

371

372

373

374

375

376

377

Knee, 16 (2009) 398-404.

[38] S. Snelling, R. Rout, R. Davidson, I. Clark, A. Carr, P.A. Hulley, A.J. Price, A gene expression study of normal and damaged cartilage in anteromedial gonarthrosis, a phenotype of osteoarthritis, Osteoarthritis \& Cartilage, 22 (2014) 334-343.

[39] L. Svensson, A. Oldberg, D. Heinegård, Collagen binding proteins, Osteoarthritis \& Cartilage, 9 Suppl A (2001) S23.

[40] E.N. Blaney Davidson, A.P.M. van Caam, P.M. van der Kraan, Osteoarthritis year in review 2016: biology, Osteoarthritis and Cartilage, 25 (2017) 175-180.

[41] Junqueira, L.C. Uchôa, Junqueira's basic histology, McGraw-Hill Medical, 2013.

[42] N. Miosge, M. Hartmann, C. Maelicke, R. Herken, Expression of collagen type I and type II in consecutive stages of human osteoarthritis, Histochemistry and cell biology, 122 (2004) 229-236.

[43] X.X. Shijin Lu, Minghua Cheng, Matrine inhibits IL-1 $\beta$-induced expression of matrix metalloproteinases by suppressing the activation of MAPK and NF-KB in human chondrocytes in vitro, International journal of clinical and experimental pathology, (2015).

[44] R.H. Brophy, B. Zhang, L. Cai, R.W. Wright, L.J. Sandell, M.F. Rai, Transcriptome comparison of meniscus from patients with and without osteoarthritis, Osteoarthritis \& Cartilage, (2017) S1063458417313730.

[45] R.K. Davidson, J.G. Waters, L. Kevorkian, C. Darrah, A. Cooper, S.T. Donell, I.M. Clark, Expression profiling of metalloproteinases and their inhibitors in synovium and cartilage, Arthritis research \& therapy, 8 (2006) R124.

[46] C. Karlsson, T. Dehne, A. Lindahl, M. Brittberg, A. Pruss, M. Sittinger, J. Ringe, Genome-wide expression profiling reveals new candidate genes associated with osteoarthritis, Osteoarthritis and cartilage / OARS, Osteoarthritis Research Society, 18 (2010) 581-592.

\section{Supplementary Tables}

Supplementary Table 1: Patient data

Supplementary Table 2: qPCR primers used in this study

Supplementary Table 3: Differentially expressed lncRNA between OA and non-OA groups

Supplementary Table 4: Differentially expressed mRNA between OA and non-OA groups

Supplementary Table 5: Biological processes corresponding to up-regulated mRNA in OA and non-OA groups, as identified through GO enrichment analysis

Supplementary Table 6: Cellular processes corresponding to up-regulated mRNA in OA and non-OA groups, as identified through GO enrichment analysis

Supplementary Table 7: Molecular functions corresponding to up-regulated mRNA in OA and non-OA groups, as identified through GO enrichment analysis

Supplementary Table 8: Biological processes corresponding to down-regulated mRNA in OA and non-OA groups, as identified through $\mathrm{GO}$ enrichment analysis

Supplementary Table 9: Cellular process corresponding to down-regulated mRNA in OA and non-OA groups, as identified through GO enrichment analysis

Supplementary Table 10: Molecular functions corresponding to down-regulated mRNA in OA and non-OA groups, as identified through GO enrichment analysis

Supplementary Table 11: Pathway analysis of up-regulated mRNA in OA and non-OA groups

Peer) reviewing PDF | (2018:04:27804:5:1:NEW 11 Jul 2019) 
378 Supplementary Table 12: Pathway analysis of down-regulated mRNA in OA and non-OA groups

379 Supplementary Table 13: Dysregulated mRNAs enriched in the PI3K-Akt signal pathway

380 Supplementary Table 14: LncRNA-mRNA interaction pairs based in the PI3K-Akt signal pathway

381 Supplementary Table 15: Top 500 lncRNA-mRNA interaction pairs in the PI3K-Akt signal pathway

382

\section{Supplementary Figures}

384 Supplementary Figure 1: Quality control of total RNA. (A) SDS-PAGE gels of total RNA; 28S ribosomal RNA and 385 18S ribosomal RNA bands appeared in all tissue samples. (B) SDS-PAGE electrophoresis pattern of total RNA. (C)

386

387

388

389

390

391

392

393

394

395 Table legend

396

397

\section{$398 \quad$ Figure legend}

399

Fig. 1. Volcano plot and hierarchical clustering of lncRNA and mRNA differential expression profiles between non-

400 OA and OA groups for 10 cartilage samples. (A) Volcano plot of dysregulated lncRNA and mRNA (dots denote

401

402 gene). Grey spots represent $p>0.05$; green spots represent $p \leq 0.05$ and fold-change $<2$. Red spots represent upregulated genes $(p \leq 0.05$ and fold-change $\geq 2.0)$, and blue spots represent up-regulated genes $(p \leq 0.05$ and fold-

403 change $\leq-2.0$ ). Heatmap of differentially expressed lncRNAs (B) and mRNAs (C). Colors denote relative

404 expression: red represents high relative expression and green represents low relative expression. 
405 Fig. 2. GO analysis of abnormal mRNAs expressed between OA and non-OA groups. Enriched GO terms targeted 406 by up-regulated mRNAs (A-C) and down-regulated mRNAs (D-F) according to biological processes, cell 407 components, and molecular function ( $p<0.05$ was statistically significant according to a Fisher's exact test).

408 Fig. 3. KEGG pathway analysis of abnormal mRNAs between OA and non-OA groups. Top 20 pathways targeted 409 by down-regulated (A) and up-regulated (B) transcripts $(p<0.05$ was statistically significant according to a Fisher's 410 exact test).

411 Fig. 4. LncRNA-mRNA co-expression network. Green node represents mRNA and red node represents lncRNA.

412 Line indicates a lncRNA-mRNA co-expression relationship. The node degree is indicated by the node size.

413 Fig. 5. QPCR and microarray data. Values are means \pm SD $(n=53)$, and ${ }^{*} p<0.05$ was statistically significant 414 compared to the non-OA group.

415 Fig. 6. mRNA levels of COL1A1 (A), COL1A2 (B) and lnc-SAMD14-4 (C) in IL-1 $\beta$-treated human primary 416 chondrocytes, as detected by qPCR. The mRNA expression was normalized to GAPDH expression and conversion 417 by $2^{-\Delta \Delta C t}$. Values are mean \pm SD $(n=3)$. Data were analyzed using one-way ANOVA, followed by Dunnett- $t$ test. 418 Fig. 7. Effect of lnc-SAMD14-4 overexpression on relative expression levels of COL1A1 (A) and COL1A2 (B) in 419 IL-1 $\beta$-treated human primary chondrocytes, as detected by qPCR. Data were analyzed using one-way ANOVA, 420 followed by Dunnett- $t$ test.

421 Fig. 8. Effect of lnc-SAMD14-4 suppression on the relative expression levels of COL1A1 (A) and COL1A2 (B) in 422 IL-1 $\beta$-treated human primary chondrocytes, as detected by qPCR. Data were analyzed using one-way ANOVA, 423 followed by Dunnett- $t$ test. 


\section{Figure 1}

Volcano plot and hierarchical clustering of IncRNA and mRNA differential expression profiles between non-OA and OA groups for 10 cartilage tissues.

(A) Volcano plot of dysregulated IncRNA and mRNA (dots denotes gene). Grey spots represent $p>0.05$; green spots represent $p<0.05$ and fold-change $<2$. Red spots represent up-regulated genes ( $p<0.05$ and fold-change $>2.0$ ), and blue spots represent up-regulated genes ( $p<0.05$ and fold-change $<-2.0$ ). Heatmap of differentially expressed IncRNA (B) and mRNA (C). Colors denote relative expression: red represents high relative expression; green represents low relative expression. 
A

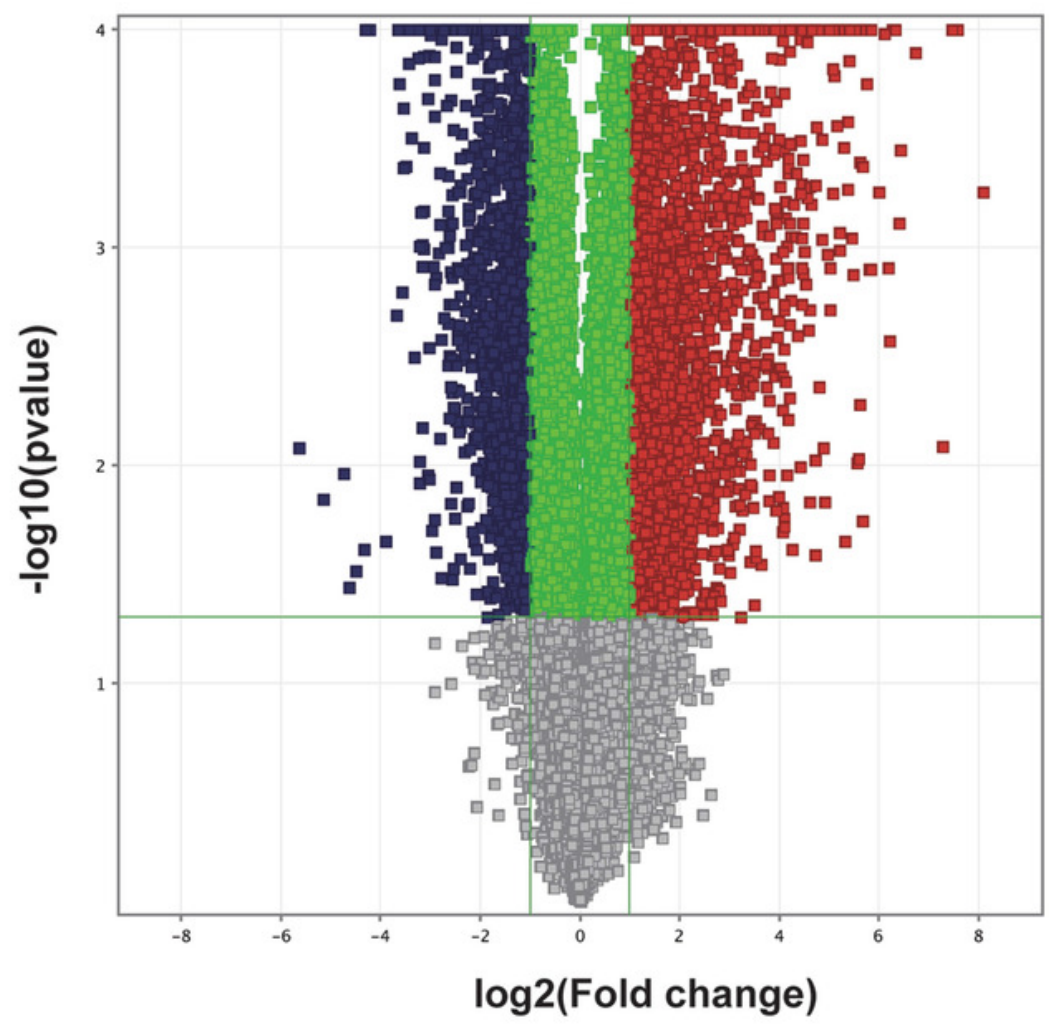

B

LncRNA

2.6
0.8
0.0
-0.6
-1.6



C

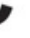


Figure 2

Go analysis of abnormal mRNAs between OA and non-OA groups.

Enriched Go terms targeted by up-regulated mRNAs (A-C) and down-regulated mRNAs (D-F) according to biological processes, cell components, and molecular function ( $p<0.05$ as statistically significant according to a Fisher's exact test). 
A

Biological Process
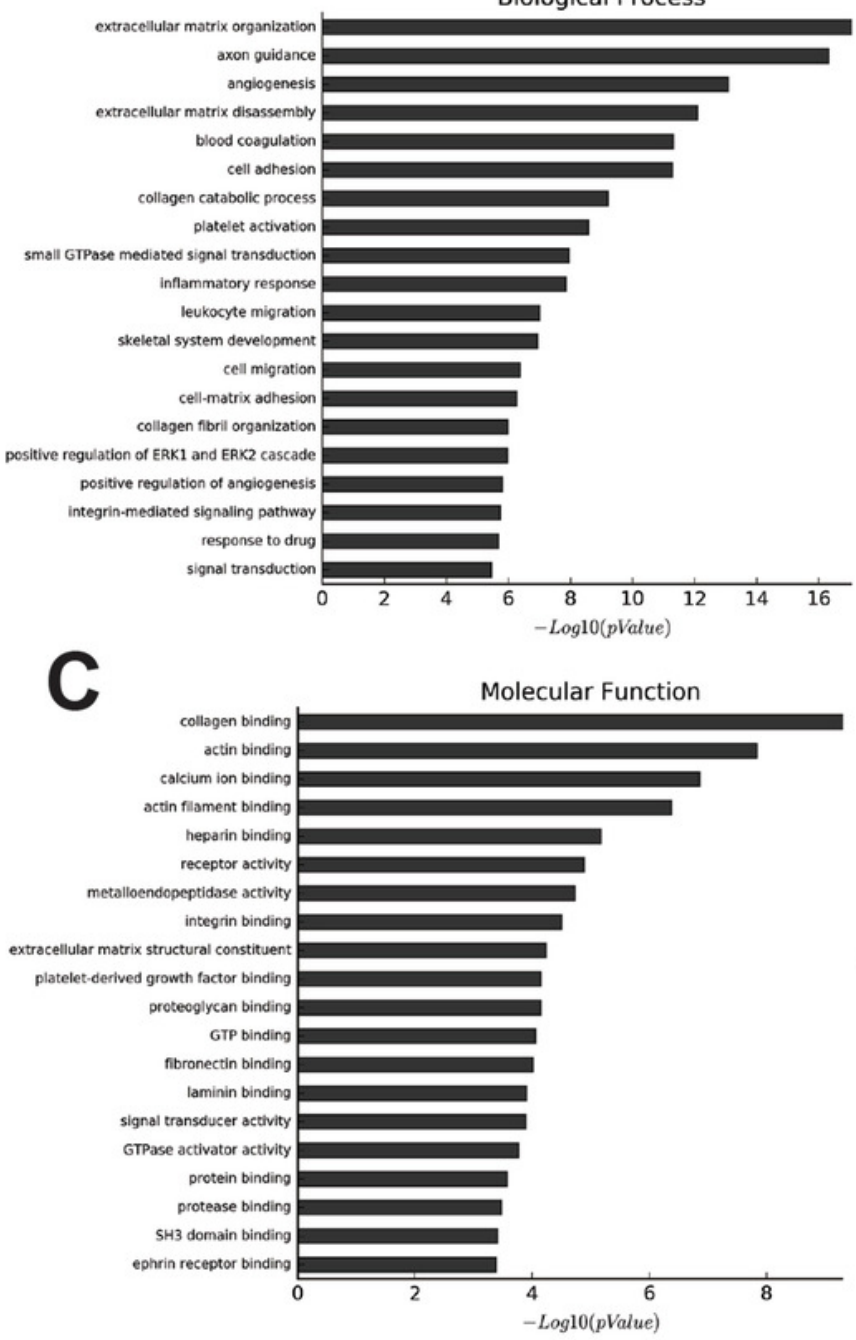

E

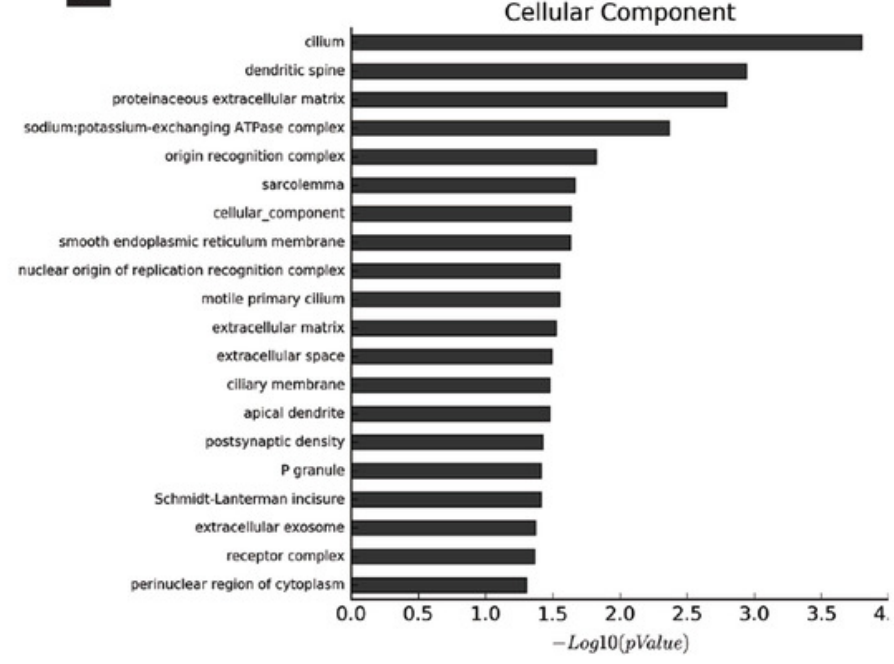

B
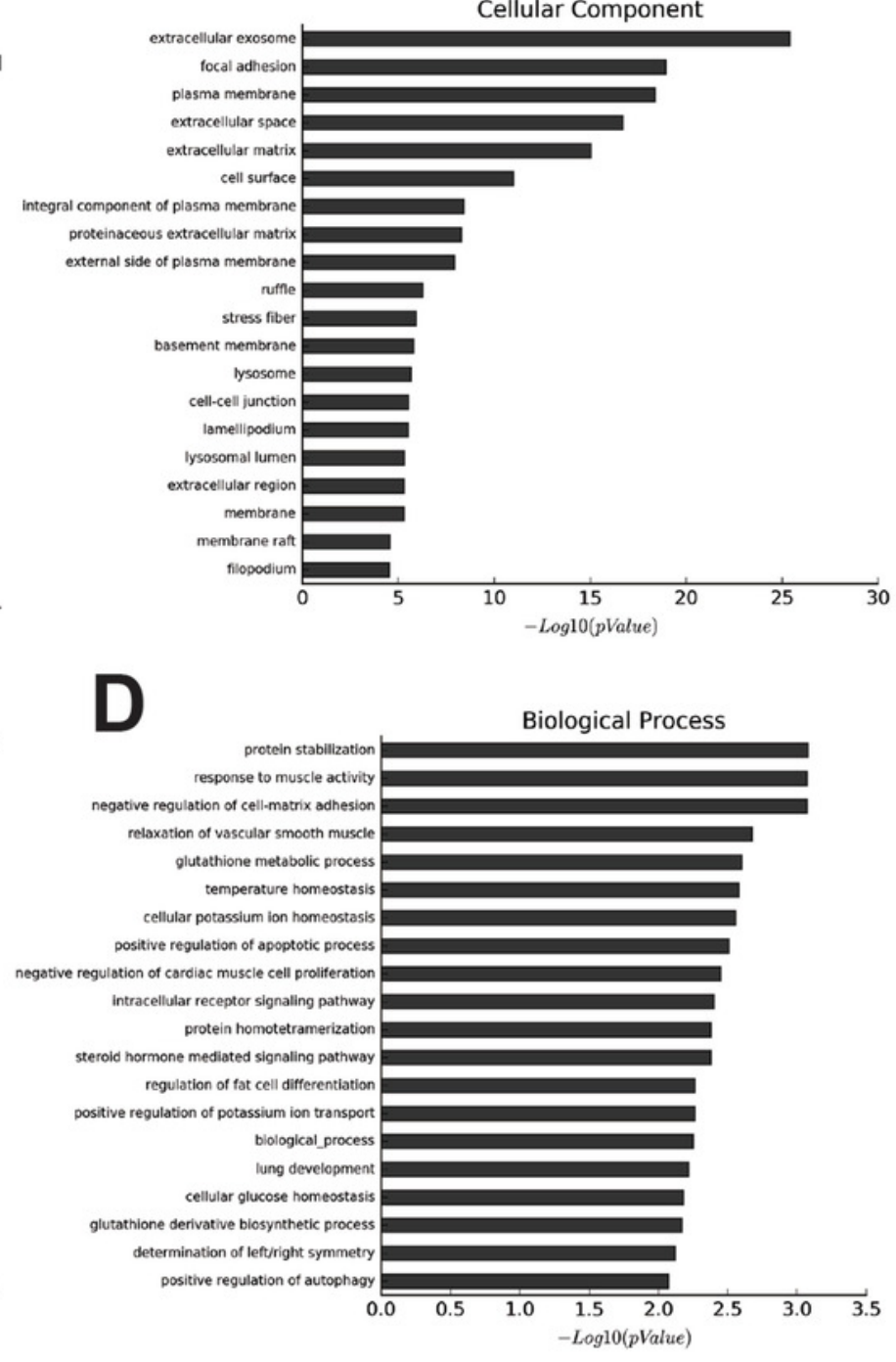

$\mathrm{F}$

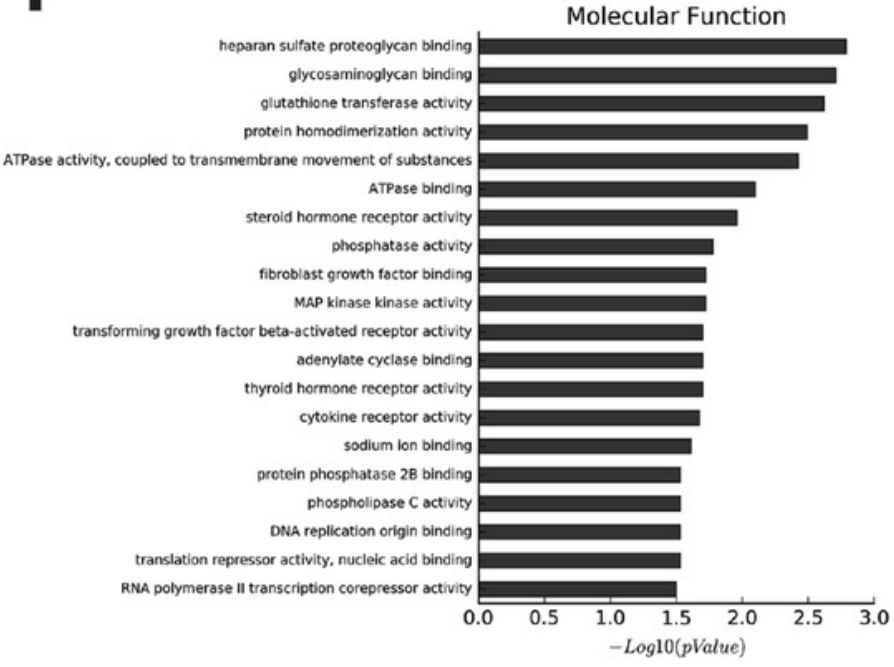




\section{Figure 3}

KEGG pathway analysis of abnormal mRNAs between OA and non-OA groups.

Top 20 pathways targeted by down-regulated $(\mathrm{H})$ and up-regulated $(\mathrm{I})$ transcripts $(p<0.05$ was statistically significant according to a Fisher's exact test).
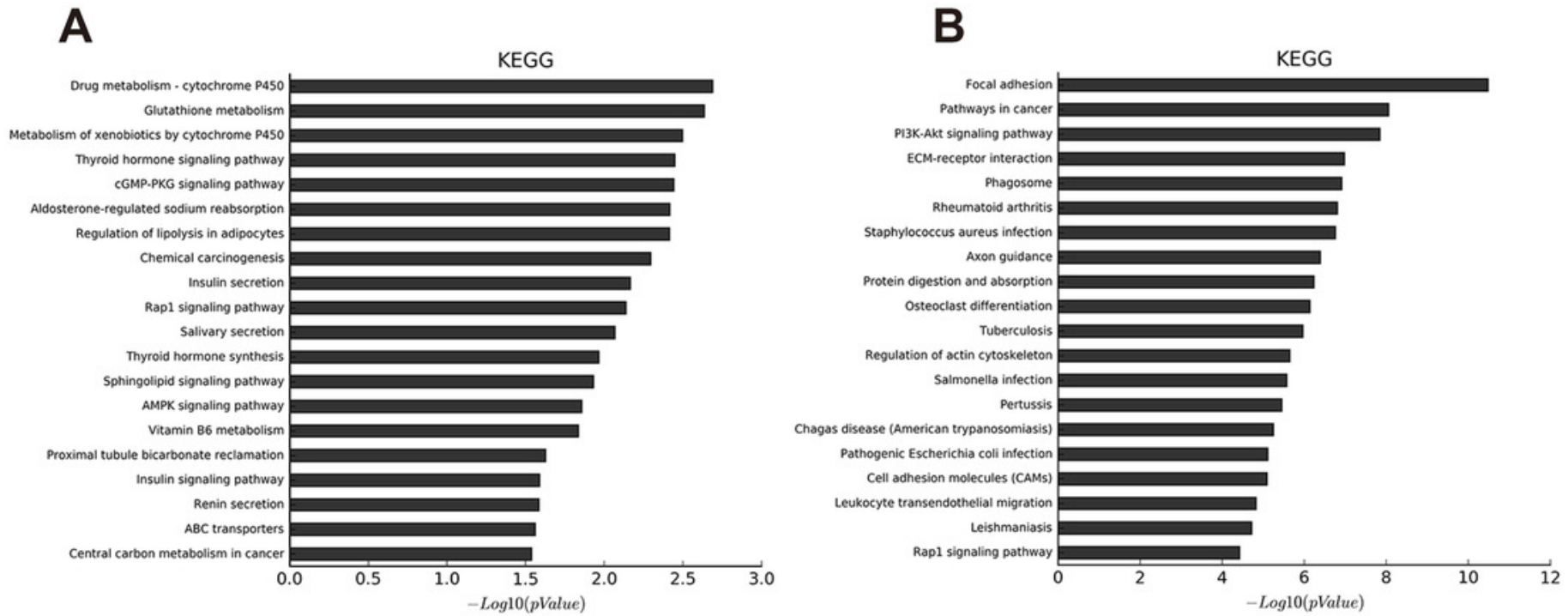
Figure 4

LncRNA-mRNA co-expression network.

Green node represents mRNA; red node represents IncRNA. Line indicates a co-expression relationship between IncRNA-mRNA. The node degree is indicated by the node size.

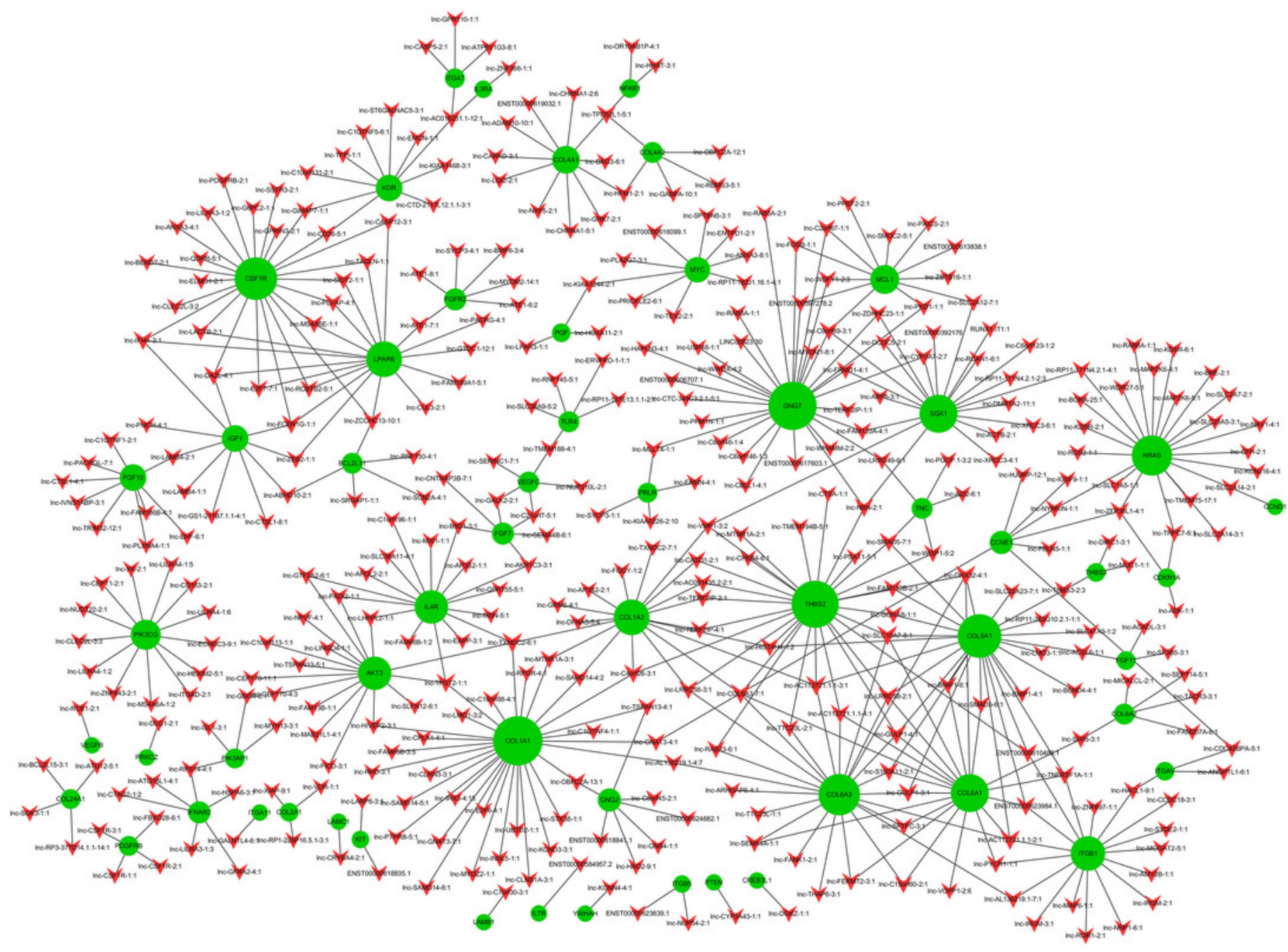


Figure 5

qPCR and microarray data.

Values are means $+S D(n=53)$, and $* p<0.05$ was statistically significant compared to the non-OA group

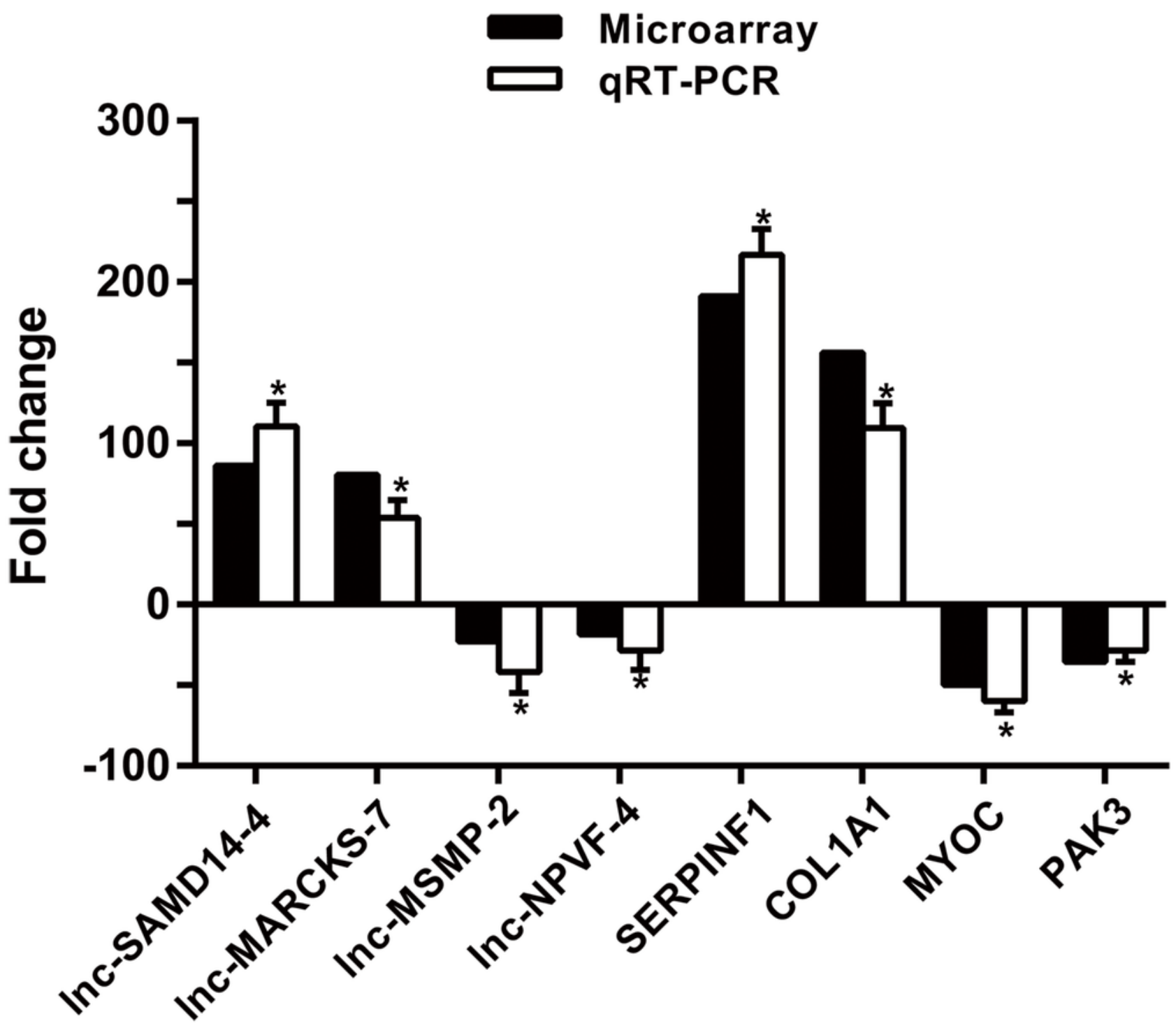


Figure 6

The mRNA levels of COL1A1 (A) $\square$ COLIA2 (B) and Inc-SAMD14-4 (C) were detected by QPCR in the IL-1 1 -treated human primary chondrocytes.

The mRNA levels of COLIA1 (A) $\square C O L 1 A 2$ (B) and Inc-SAMD14-4 (C) were detected by QPCR in the IL-1 $\beta$-treated human primary chondrocytes. The mRNA expression was normalized to GAPDH expression and conversion by $2^{-\Delta \Delta C t}$. Values are means $+S D(n=3)$. Data were analyzed using one-way ANOVA followed by Dunnett-t test.
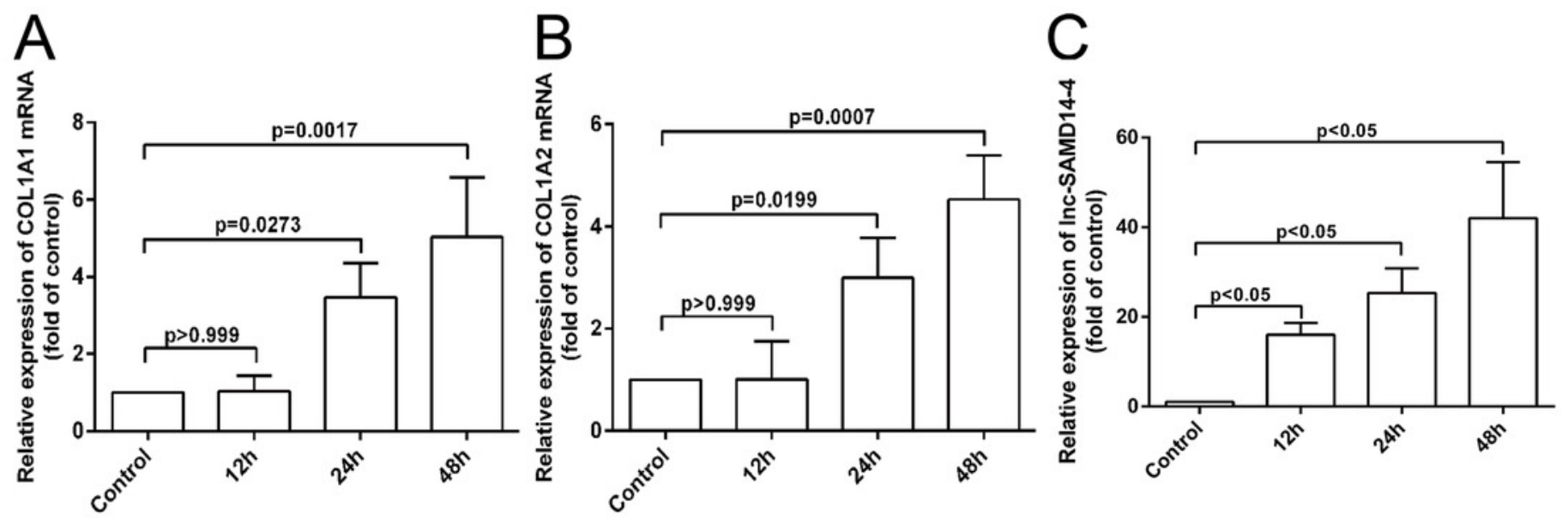
Figure 7

Effects of Inc-SAMD14-4 overexpression on the relative expression levels of COLIA1 (A) $\square C O L 1 A 2(B)$ in IL-13-treated human primary chondrocytes detected by QPCR.

Effects of Inc-SAMD14-4 overexpression on the relative expression levels of COLIAI

(A) $\square C O L 1 A 2$ (B) in IL-1 3 -treated human primary chondrocytes detected by QPCR. Data were analyzed using one-way ANOVA followed by Dunnett-t test.
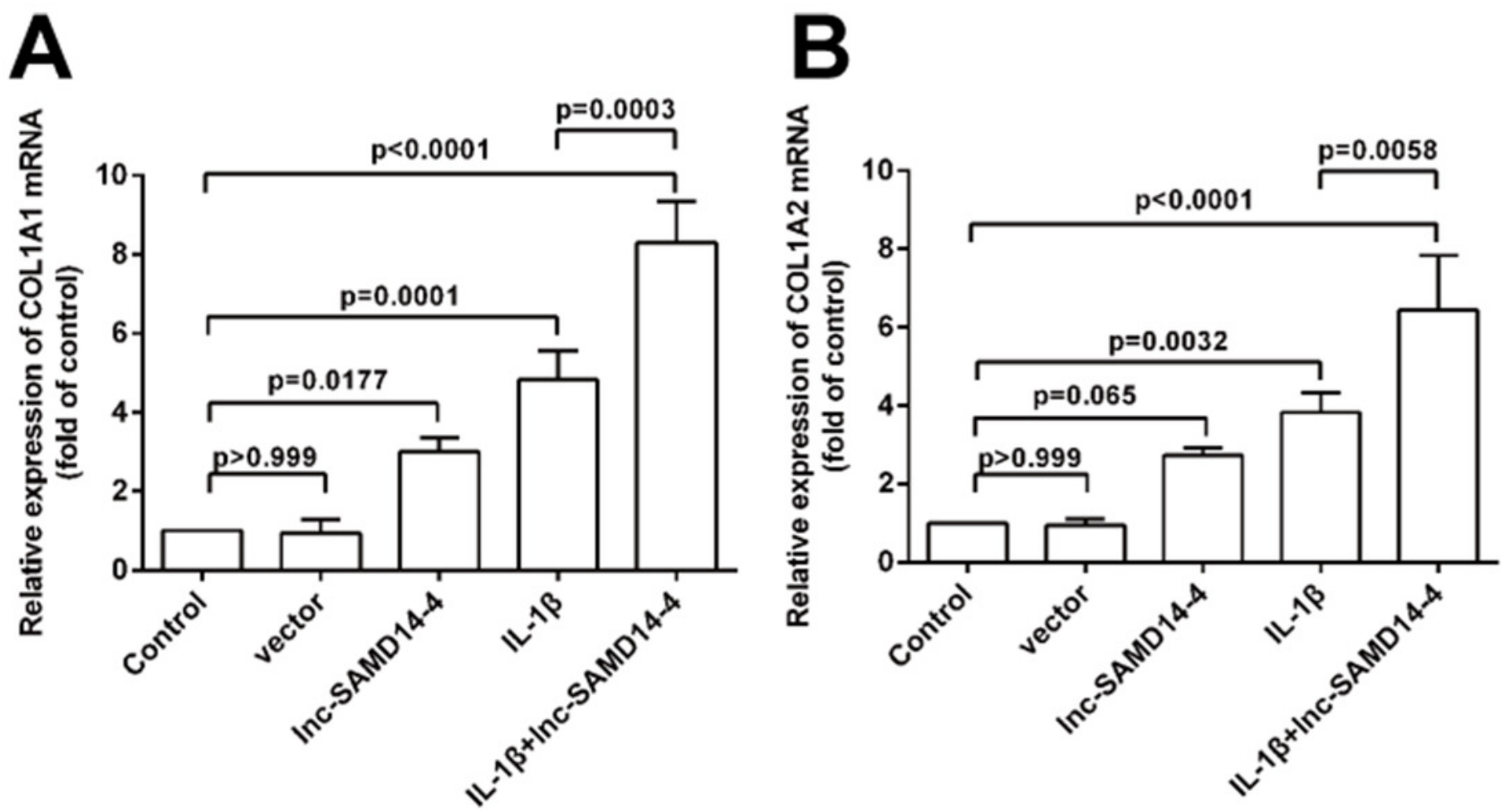
Figure 8

Effects of Inc-SAMD14-4 suppression on the relative expression levels of COL1A1 (A) $\square C O L 1 A 2(B)$ in IL-1 $\beta$-treated human primary chondrocytes detected by QPCR.

Effects of Inc-SAMD14-4 suppression on the relative expression levels of COL1A1

(A) $\square C O L 1 A 2$ (B) in IL-1ß-treated human primary chondrocytes detected by QPCR. Data were analyzed using one-way ANOVA followed by Dunnett-t test.
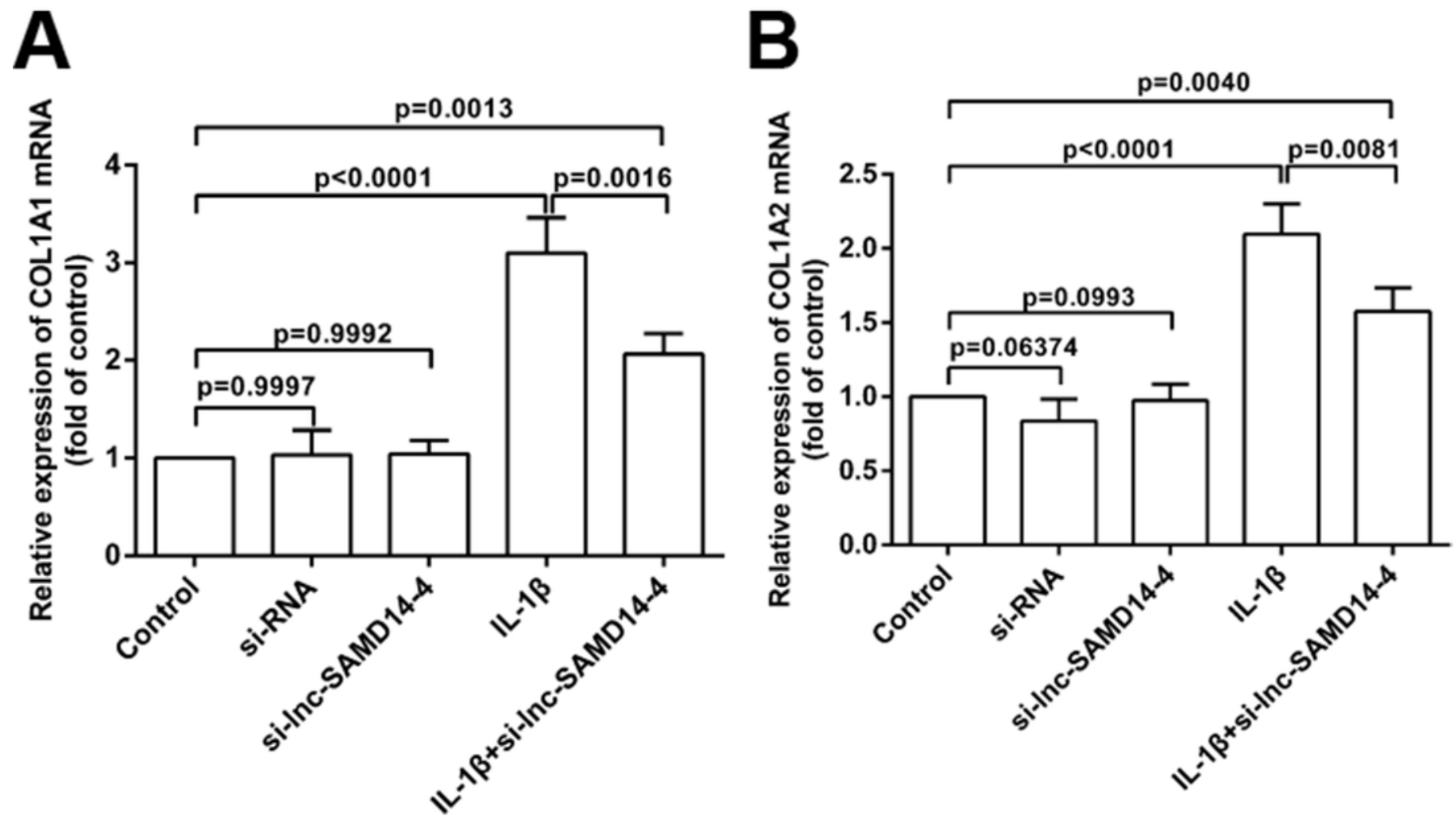


\section{Table 1 (on next page)}

Most regulated IncRNAs in this study 


\begin{tabular}{|c|c|c|c|c|}
\hline GeneSymbol & Regulation & Type & Fold change(abs) & $P$-value \\
\hline lnc-SAMD14-4 & $\mathrm{Up}$ & Exonic & 86.11047 & 0.00036 \\
\hline lnc-MARCKS-7 & Up & ------- & 80.51785 & 0.0000923 \\
\hline lnc-SAMD14-5 & Up & Exonic & 76.39162 & 0.0000161 \\
\hline $\operatorname{lnc}-A P 3 S 2-2$ & Up & Exonic & 57.44485 & 0.001265177 \\
\hline lnc-CASD1-2 & Up & Exonic & 56.14244 & 0.001261528 \\
\hline lnc-TSPAN13-4 & $\mathrm{Up}$ & Exonic & 50.915787 & 0.00000633 \\
\hline lnc-PPM1N-1 & Up & Exonic & 47.80361 & 0.009312962 \\
\hline lnc-TRPC4-2 & Up & Exonic & 47.43022 & 0.009825662 \\
\hline lnc-C1 orf196-1 & Up & Linc & 40.125366 & 0.0000202 \\
\hline lnc-SMAD5-7 & Up & Exonic & 39.585285 & 0.022567188 \\
\hline lnc-TSPAN13-5 & $\mathrm{Up}$ & Exonic & 37.4391 & 0.00000077 \\
\hline lnc-SFRP1-2 & Up & Exonic & 36.96935 & 0.001035282 \\
\hline lnc-HJURP-12 & Up & Exonic & 36.914413 & 0.000862 \\
\hline lnc-TEX2-2 & Up & Exonic & 33.92366 & 0.000572 \\
\hline lnc-MS4A6E-1 & Up & Exonic & 31.000843 & 0.001087416 \\
\hline lnc-MSMP-2 & Down & Exonic & 22.78652 & 0.030858 \\
\hline lnc-NPVF-4 & Down & Linc & 18.66038 & 0.0000169 \\
\hline lnc-CBLL1-4 & Down & Exonic & 14.84985 & 0.022533 \\
\hline MLLT4-AS1 & Down & ------- & 12.8248 & 0.002052 \\
\hline lnc-NPVF-10 & Down & ------- & 12.68418 & 0.0000278 \\
\hline lnc-ASZ1-3 & Down & Exonic & 11.32834 & 0.000425 \\
\hline SAMD12 & Down & ------- & 9.023141 & 0.006789 \\
\hline lnc-NPVF-5 & Down & ------- & 9.018275 & 0.000095 \\
\hline lnc-IRGM-3 & Down & Exonic & 8.909008 & 0.001227 \\
\hline lnc-ERCC5-4 & Down & Linc & 8.883492 & 0.000686 \\
\hline lnc-SSR3-6 & Down & & 8.876818 & 0.000349 \\
\hline lnc-DLGAP1-9 & Down & Exonic & 8.776601 & 0.001021 \\
\hline lnc-SSR3-5 & Down & Antisense & 8.354682 & 0.000208 \\
\hline lnc-CHI3L1-1 & Down & Exonic & 8.350381 & 0.011126 \\
\hline
\end{tabular}




\begin{tabular}{|c|c|c|c|c|}
\hline lnc-OXNAD1-6 & Down & Antisense & 8.14148 & 0.0000128 \\
\hline
\end{tabular}


Table 2 (on next page)

Most regulated mRNAs in this study 


\begin{tabular}{|c|c|c|c|}
\hline GeneSymbol & Regulation & Fold change(abs) & P-value \\
\hline MMP13 & $\mathrm{Up}$ & 272.535 & 0.000564 \\
\hline SERPINF1 & $\mathrm{Up}$ & 191.17508 & 0.000000141 \\
\hline COL1A1 & $\mathrm{Up}$ & 175.43033 & 0.0000199 \\
\hline POSTN & $\mathrm{Up}$ & 156.12422 & 0.008303139 \\
\hline LRRC15 & $\mathrm{Up}$ & 105.57993 & 0.000129 \\
\hline COL1A2 & $\mathrm{Up}$ & 85.32694 & 0.000776 \\
\hline THBS2 & $\mathrm{Up}$ & 74.47372 & 0.002686227 \\
\hline FNDC1 & Up & 72.167336 & 0.001257106 \\
\hline RP11-429G19.3 & Up & 69.320656 & 0.000105 \\
\hline MS4A4A & $\mathrm{Up}$ & 64.19533 & 0.000564 \\
\hline FPR3 & Up & 56.89943 & 0.0000552 \\
\hline MSR1 & $\mathrm{Up}$ & 53.758953 & 0.000178 \\
\hline AHR & $\mathrm{Up}$ & 52.798218 & 0.0000266 \\
\hline TNFAIP6 & $\mathrm{Up}$ & 51.144848 & 0.018054951 \\
\hline PLXDC1 & $\mathrm{Up}$ & 51.0092 & 0.00043 \\
\hline MYOC & Down & 49.693558 & 0.008422094 \\
\hline PAK3 & Down & 35.395626 & 0.014492353 \\
\hline APOD & Down & 26.913952 & 0.011042964 \\
\hline MSMP & Down & 24.774714 & 0.036745384 \\
\hline SLC26A4 & Down & 20.350153 & 0.024579711 \\
\hline AC003090.1 & Down & 19.897524 & 0.0001 \\
\hline CYP4F11 & Down & 12.0151615 & 0.001603253 \\
\hline CTTNBP2 & Down & 11.738498 & 0.000434 \\
\hline ABCA5 & Down & 10.876956 & 0.000144 \\
\hline ZMAT1 & Down & 9.586397 & 0.000137 \\
\hline
\end{tabular}

1 